\title{
TRANSPLANTE RENAL NO PACIENTE DIABÉTICO COM DOENÇA RENAL CRÔNICA
}

\author{
Kidney transplantation in diabetic patients with chronic kidney disease
}

\author{
Irene de Lourdes Noronha',2, Pablo Girardelli Mendonça Mesquita1,2, José Osmar Medina Pestana ${ }^{3}$
}

\begin{abstract}
RESUMO
O diabetes mellitus é uma das principais etiologias da doença renal crônica no Brasil e no mundo. Nos pacientes que se encontram em estágio $\mathrm{V}$ de doença renal crônica, torna-se necessária a terapia renal substitutiva, que pode ser realizada através do transplante renal ou diálise. A diálise é a opção terapêutica com maior número de pacientes, apesar de ser a modalidade associada à menor sobrevida do paciente. As opções de transplante para paciente diabético são o transplante renal com doador vivo ou falecido ou transplante duplo de rim e pâncreas. O presente estudo teve como objetivo fazer uma revisão dos aspectos específicos do transplante renal em pacientes diabéticos, avaliar o impacto do transplante renal nas complicações crônicas do diabetes e, por fim, apresentar os resultados disponíveis na literatura que permitem avaliar qual a melhor modalidade de transplante para esses doentes. Com relação à avaliação pré-transplante, devido ao alto risco cardiovascular, a avaliação da doença arterial coronariana e doença arterial periférica devem ser realizadas de forma mais detalhada no paciente diabético. Além disso, o estudo das disfunções miccionais está indicado, devido à alta prevalência de bexiga neurogênica nesses pacientes. Em relação às complicações crônicas do diabetes, as evidências atuais sugerem que no transplante renal ocorre uma estabilização das lesões da retina na maioria dos pacientes, melhora na qualidade de vida, e melhora dos episódios de hipotensão postural e sintomas gastrointestinais. Em contrapartida, observa-se maior número de eventos cardiovasculares em comparação aos não-diabéticos e recidiva da nefropatia em porcentagem limitada dos casos. Com relação às modalidades de transplante disponíveis para o paciente diabético, os melhores resultados de sobrevida do paciente e do enxerto foram obtidos através da realização de transplante renal isolado com doador vivo ou com transplante duplo de rim e pâncreas, sendo o transplante renal isolado com doador falecido a modalidade associada aos piores índices de sobrevida, tanto do paciente quanto do enxerto.
\end{abstract}

Descritores: Diabetes Mellitus; Insuficiência Renal; Transplante.

\footnotetext{
Instituição:

${ }^{1}$ Laboratório Nefrologia Celular, Genética e Molecular da Faculdade de Medicina da Universidade de São Paulo, São Paulo, SP, Brasil

2 Departamento de Nefrologia (hemodiálise e transplante) do Hospital Beneficência Portuguesa, São Paulo, SP, Brasil

${ }_{3}^{3}$ Departamento de Nefrologia do Hospital do Rim e Hipertensão - Universidade Federal de São Paulo, São Paulo, SP, Brasil
}

Correspondência:

Profa. Dra. Irene L. Noronha

Laboratório de Nefrologia Celular, Genética e Molecular da Faculdade de Medicina da USP

Av. Dr. Arnaldo, 455, $4^{\circ}$ andar, Lab 4304, CEP: 01246-903, São Paulo, SP, Brasil

Telefone: (11) 3061-8403.

E-mail: irenenor@usp.br

Recebido em: 22.07.2010

Aceito em: 01.09.2010

\section{INTRODUÇÃo}

O diabetes mellitus é uma das principais causas da doença renal crônica no Brasil e no mundo. A história natural da nefropatia diabética caracteriza-se pela evolução lenta e progressiva, culminando com doença renal crônica estágio $\mathrm{V}$, que leva à necessidade de terapia substitutiva renal (diálise e/ou transplante).

Os pacientes diabéticos submetidos a tratamento dialítico apresentam maior mortalidade em relação ao transplante renal, como será discutido adiante.

O transplante renal em pacientes diabéticos possui algumas particularidades tanto no pré-transplante como no pós-transplante. No pré-transplante destaca-se a importância da avaliação criteriosa do risco cardiovascular, da doença vascular periférica e das disfunções miccionais. No pós-transplante ocorre maior dificuldade de controle glicêmico, aumento de incidência de infecções, além de maior risco de eventos cardiovasculares.

Neste capítulo serão abordados vários aspectos específicos do transplante renal em paciente diabético. 


\section{MATERIAIS E MÉTODOS}

O trabalho consiste de revisão bibliográfica realizado através de pesquisa dos bancos de dados MEDLINE (PubMed: Cumulative Index Medicus). Foram selecionados artigos científicos publicados preferencialmente nos artigos mais recentes. Durante o levantamento bibliográfico foram utilizadas diversas combinações de palavras-chaves, como por exemplo: "diabetes mellitus", "nefropatia diabética", "transplante renal", "transplante duplo rim e pâncreas" e "insuficiência renal”. A pesquisa bibliográfica incluiu artigos de revisão, artigos originais e consensos.

\section{O TRANSPLANTE RENAL NO PACIENTE DIABÉTICO}

O transplante renal é uma modalidade de transplante bem estabelecida. Desde a década de 60, quando a técnica foi implantada no nosso meio até os dias de hoje, mais de 45.000 transplantes de rim já foram realizados no Brasil. ${ }^{1}$

O transplante renal pode ser feito com doador vivo ou doador falecido. Trata-se de uma cirurgia de média duração (de três a quatro horas), sem necessidade de abordagem intraperitoneal, o que facilita a rápida recuperação no pós-operatório. Além desses aspectos cirúrgicos e, graças à experiência clínica acumulada ao longo dos 45 anos de atividade nessa área, os resultados do transplante renal são muito bons. ${ }^{2}$ Além do tempo de internação do transplante renal ser relativamente curto, deve-se ressaltar que a necessidade de reinternações é limitada.

No Brasil existem 235 equipes cadastradas para realizar transplante renal, sendo que 136 encontram-se ativas. 1 Esses centros estão habilitados para realizar transplante renal em pacientes com doença renal crônica em estágio $\mathrm{V}$, incluindo pacientes diabéticos. Já para realização de transplante de pâncreas, há necessidade de equipe especificamente treinada para esse procedimento. No Brasil existem apenas 43 equipes cadastradas para realizar transplante de pâncreas, sendo que apenas 20 estão ativas. ${ }^{1}$

Dessa forma, o transplante de rim isolado constitui uma importante alternativa de terapia substitutiva da função renal para pacientes diabéticos com insuficiência renal crônica terminal. A comparação dos resultados do transplante renal em pacientes diabéticos com outras modalidades de tratamento será abordada mais adiante neste capítulo.

\section{Indicação de transplante renal: abordagem pré-emptiva}

O transplante renal pode ser realizado quando o paciente já se encontra realizando regularmente diálise crônica ou antes de iniciar tratamento dialítico, chamado transplante pré-emptivo.

De acordo com as diretrizes elaboradas pelo KDOQI (Kidney Disease Outcomes Quality Initiative), pacientes com insuficiência renal devem ser encaminhados ao nefrologista quando o clearance de creatinina estiver abaixo de $30 \mathrm{ml} / \mathrm{min} / 1,73 \mathrm{~m}^{2} .{ }^{3} \mathrm{O}$ encaminhamento do paciente nessa fase está associado a uma menor mortalidade e menor velocidade de progressão da doença renal crônica. ${ }^{5}$ Além disso, à medida que a doença evolui, o médico pode esclarecer o paciente a respeito dos diversos tipos de tratamento e já prepará-lo para a diálise e/ou transplante.

Apesar de não existirem estudos controlados, há evidências clínicas de que pacientes diabéticos em fases avançadas da doença renal crônica beneficiam-se com o início precoce da terapia substitutiva renal, quer seja diálise ou transplante, denominada pré-emptiva. ${ }^{5}$ Ainda não existem dados na literatura que permitam definir a faixa adequada de comprometimento da função renal para indicar a terapia pré-emptiva, porém a maioria dos relatos considera a faixa de clearance de creatinina entre 15 e $20 \mathrm{ml} / \mathrm{min} / 1,73 \mathrm{~m}^{2}$ para indicar o tratamento pré-emptivo.

Nos relatos da literatura em que o transplante renal foi realizado de forma pré-emptiva são descritos melhores resultados na sobrevida de pacientes diabéticos tanto do tipo 1 como do tipo $2 .^{5}$ A análise de 23.000 pacientes diabéticos do registro Scientific Registry of Transplant Recipients submetidos a transplante renal revelou que $14,4 \%$ dos pacientes com DM tipo 1 e $6,7 \%$ dos pacientes com DM tipo 2 realizaram o transplante de forma pré-emptiva. Foi observada uma diminuição significativa no risco de mortalidade, sendo o risco relativo de 0,57 para DM tipo 1 e 0,65 para DM tipo 2 .

\section{Avaliação pré-transplante renal}

A avaliação pré-transplante do paciente diabético apresenta vários pontos em comum com a do não-diabético. Em toda avaliação de receptores candidatos a transplante renal recomenda-se a avaliação de infecções ativas, doenças cardiovasculares, pulmonares, hepáticas, entre outras, que são comuns a pacientes diabéticos e não-diabéticos. Porém, devido ao fato dos pacientes diabéticos com doença renal crônica terem uma tendência maior a desenvolver aterosclerose precoce (com maior prevalência de eventos como infarto agudo do miocárdio, acidente vascular cerebral e eventos vasculares periféricos) ${ }^{6}$ é importante a avaliação cuidadosa da doença vascular.

O paciente diabético apresenta dois tipos de doença vascular: macroangiopatia e microangiopatia. A macroangiopatia diabética caracteriza-se por lesões ateroscleróticas das coronárias, carótidas, aorta e outros vasos periféricos, enquanto a disfunção microcirculatória não-oclusiva envolve capilares e arteríolas, principalmente de rins, retina e nervos periféricos.

A aterosclerose do diabético é mais difusa, mais grave e se manifesta em idade mais precoce do que a aterosclerose do paciente nãodiabético. ${ }^{6}$ A calcificação da placa intimal e da camada média (esclerose de Mönckeberg) é característica da aterosclerose diabética. ${ }^{7}$

\section{Avaliação cardiovascular}

As complicações cardiovasculares nos pacientes diabéticos são consequência da doença aterosclerótica que, nesses pacientes, além de grave apresenta uma evolução particularmente acelerada. ${ }^{6}$ Os fatores de risco para o desenvolvimento da doença cardiovascular podem ser divididos em fatores de risco tradicionais (hipertensão arterial, sexo masculino, tabagismo, história familiar, diabetes, obesidade, sedentarismo, idade $>55$ anos em homens e $>65$ anos em mulheres, doença renal crônica) e fatores relacionados à uremia. ${ }^{8}$

Pacientes diabéticos em tratamento dialítico têm prevalência aumentada de doença arterial coronariana. ${ }^{9}$ A doença coronariana é freqüentemente tri-arterial e difusa, acometendo porções proximais e distais das artérias.

A doença coronariana em diabéticos pode ter curso assintomático 
em $40 \%$ dos pacientes,$^{10}$ o que dificulta seu reconhecimento, e, por esse motivo, tem impacto negativo na evolução clínica. Cerca de 1/3 dos pacientes diabéticos tipo 1 com doença renal crônica assintomáticos já apresentam estenoses coronarianas significativas ao exame de coronariografia. ${ }^{11}$ A manifestação isquêmica silenciosa pode ser consequência da neuropatia diabética, que compromete a resposta autonômica.

A angiocoronariografia continua sendo o melhor método para a identificação da doença arterial coronariana. ${ }^{12}$ No entanto, além do custo mais alto, apresenta riscos inerentes ao procedimento, principalmente em pacientes diabéticos em fase pré-dialítica, situação onde o uso de contraste radiológico pode acelerar a necessidade de diálise. Por esse motivo, exames não-invasivos como ecocardiografia com dobutamina e cintilografia miocárdica com dipiradamol, apesar de apresentarem menor sensibilidade e especificidade para a identificação da doença arterial coronariana, podem ser considerados uma alternativa.

As diretrizes da Sociedade Americana de Transplante recomendam que pacientes diabéticos com angina candidatos a transplante devem realizar diretamente a angiocoronariografia. No caso de pacientes assintomáticos, a indicação de angiocoronariografia é questionável. Nesses casos, exames não-invasivos podem ser indicados como primeira avaliação. No entanto, quando esses testes são positivos ou duvidosos, a arteriografia coronariana está indicada. ${ }^{13}$

Em pacientes diabéticos com doença renal crônica que ainda não se encontram em diálise e que têm indicação de realizar angiocoronariografia, deve-se tentar manobras para redução do risco da nefropatia por contraste, como hidratação endovenosa e administração de n-acetilcisteína ou solução com bicarbonato. ${ }^{14}$

Cirurgias de revascularização miocárdica ou angioplastia devem, preferencialmente, ser realizadas antes do transplante. O uso de antiagregantes plaquetários como o clopidogrel impede a realização do transplante durante o período de sua administração, devendo ser suspenso 5 dias antes da realização do transplante com doador vivo. ${ }^{15}$ Por outro lado, o uso de aspirina, que é mantido indefinidamente, pode aumentar o risco de sangramento durante o transplante, mas não implica em contra-indicação para o procedimento cirúrgico.

Avaliação da doença cerebrovascular

O diabetes é um fator de risco independente para desenvolvimento de acidente vascular cerebral isquêmico. Pacientes diabéticos apresentam risco duas vezes maior para isquemia cerebral que pacientes não diabéticos. ${ }^{16}$

A principal ferramenta para investigar a presença de estenoses significativas das artérias carótidas é o doppler de carótidas e artérias vertebrais. Havendo lesões significativas, exames de imagens com contraste radiológico devem ser realizados, considerando-se os mesmos aspectos descritos acima para doença cardiovascular.

\section{Avaliação da doença vascular periférica}

A aterosclerose disseminada dos membros inferiores, comum em diabéticos, pode não ter manifestações isquêmicas evidentes, graças à compensação que ocorre pela circulação colateral. Pacientes diabéticos apresentam maior prevalência de doença vascular periférica, sendo de 10,8\% em diabéticos, comparada a 3,6\% em não-diabéticos. ${ }^{17} \mathrm{Com}$ a associação de diabetes e doença renal crônica, o risco do paciente apresentar doença vascular periférica aumenta de duas a três vezes. ${ }^{17}$

A avaliação da doença vascular periférica inicia-se com detalhada história clínica e exame físico, com especial atenção à palpação de pulsos periféricos, além da busca de possíveis lesões periféricas (alteração da coloração do membro, lesões tróficas e úlceras). Para o diagnóstico e caracterização da doença vascular periférica, recomenda-se a realização de doppler dos vasos ilíacos e dos vasos dos membros inferiores e, se necessário, complementado por exame de imagem com contraste.

A realização do exame com contraste radiológico do tipo angiografia, angiotomografia ou angiorressonância apresenta limitações em pacientes candidatos a transplante com doença renal crônica. Nos exames com contraste iodado, que é nefrotóxico mesmo em doses baixas e mesmo em formulações não-iônicas de baixa osmolaridade ou iso-osmolaridade, ${ }^{14}$ pode ocorrer piora da função renal após o exame. Quando a realização do exame é imperativa, deve-se proceder às manobras profiláticas descritas acima.

A realização de angiorressonância também é limitada em pacientes com doença renal crônica com clearance de creatinina abaixo de $30-60 \mathrm{ml} / \mathrm{min} / 1,73 \mathrm{~m} 2$, devido ao alto risco de desenvolver fibrose sistêmica nefrogênica pelo gadolíneo. ${ }^{18}$

A associação de vasculopatia com a neuropatia diabética representa uma combinação fatal para formação de úlcera nos pés, com alta probabilidade de complicar com infecção secundária, caracterizando o pé diabético. Cerca de $25 \%$ dos diabéticos apresentam ao menos um episódio de úlcera no pé, ${ }^{19}$ sendo fator significativo para amputação. A vasculopatia diabética é responsável por $50 \%$ dos casos de amputação de membros inferiores, gerando um risco 40 vezes maior de amputações quando comparado a pacientes não diabéticos. ${ }^{20}$

\section{Avaliação urológica}

Cerca de $80 \%$ dos pacientes diabéticos são acometidos por complicações do trato urinário inferior. ${ }^{21}$ Nos pacientes diabéticos, o desenvolvimento de neuropatia autonômica no trato urinário é conhecido como bexiga neurogênica. $\mathrm{O}$ acometimento neuronal pelo diabetes no trato urinário leva à alteração da percepção sensorial de plenitude vesical, diminuição da amplitude das contrações vesicais e descoordenação esfincteriana que podem alterar o processo de enchimento e esvaziamento vesical. A disfunção vesical associada ao diabetes determina aumento do volume residual pós-miccional. ${ }^{21}$ Como conseqüência, pacientes diabéticos apresentam maior prevalência de bacteriúria assintomática e maior incidência de infecção urinária. ${ }^{22}$

Durante a realização da anamnese de paciente diabético candidato a transplante renal, recomenda-se investigar o número de infecções no último ano e os possíveis sintomas urinários relacionados com a disfunção miccional. São de fundamental importância a identificação e orientação terapêutica em casos de bexiga neurogênica na fase pré-transplante renal. $\mathrm{O}$ estudo urodinâmico deve ser considerado em pacientes com suspeita de bexiga neurogênica. ${ }^{23}$ Quando o resíduo pós-miccional é elevado, deve ser instituído regime de cateterismo intermitente.

Nos pacientes que apresentam infecções urinárias recorrentes, preconiza-se a realização de uretrocistografia miccional. ${ }^{23}$ 


\section{A cirurgia de transplante renal}

A cirurgia de transplante renal já é realizada há vários anos, com técnica bem definida e com baixo índice de complicações. Em geral, o procedimento é semelhante em diabéticos e não-diabéticos. Recomenda-se que o transplante renal isolado em pacientes diabéticos seja feito na fossa ilíaca esquerda, deixando intacta a fossa ilíaca direita para eventual transplante de pâncreas no futuro.

Sob o ponto de vista cirúrgico do transplante, a aterosclerose diabética acompanhada de calcificação vascular pode representar uma dificuldade extra para realização das anastomoses, e obriga em alguns casos a modificações técnicas.

No período pós-transplante imediato, pacientes diabéticos tendem a apresentar maior incidência de complicações cirúrgicas, como deiscência de sutura, infecção de ferida operatória e hematoma da ferida operatória. ${ }^{24}$

\section{Imunossupressão}

As bases da imunossupressão para profilaxia de rejeição em transplante renal utilizada em pacientes diabéticos não diferem dos casos de pacientes não-diabéticos. Consistem em terapia de indução, de acordo com indicações estabelecidas e terapia de manutenção, composta basicamente por um esquema tríplice composto por inibidor de calcineurina, uma droga antiproliferativa e corticóide.

Ambos inibidores de calcineurina, a ciclosporina ou o tacrolimo têm sido utilizados no esquema de imunossupressão pós-transplante. Doses um pouco mais altas de inibidores de calcineurina têm sido empregadas em pacientes diabéticos, para que sejam alcançados os níveis sanguineos preconizados. $\mathrm{O}$ tacrolimo é o inibidor de calcineurina preferencialmente usado na grande maioria dos Centros de Transplante. No caso particular de pacientes diabéticos que apresentam elevado risco cardiovascular, seu efeito benéfico na hiperlipidemia e na pressão arterial comparado à ciclosporina constitui uma vantagem adicional. ${ }^{25}$

Dentre as drogas antiproliferativas, os principais aspectos a serem considerados relacionam-se com a complicação de neuropatia autonômica diabética no trato gastrointestinal. Nesse contexto, deve-se ressaltar que a intolerância ao micofenolato devido ao desencadeamento de diarréia leva à necessidade de redução de dose ou até mesmo de suspensão da droga, com maior risco de rejeição ao enxerto. ${ }^{26}$

Os inibidores do sinal de proliferação (sirolimo e everolimo), apesar de aumentarem a resistência à insulina ${ }^{27}$, podem ser utilizados no esquema de imunossupressão de pacientes diabéticos. Uma das grandes vantagens do uso dessa classe de imunossupressores é permitir a retirada com segurança do inibidor de calcineurina ou da droga antiproliferativa. ${ }^{28,29}$ Essa intervenção se mostrou segura também em pacientes submetidos a transplante duplo, como demonstrado pelo nosso grupo em 2008. ${ }^{30}$

Os corticóides, pelo fato de contribuírem para o desenvolvimento da hiperlipidemia e hipertensão arterial, que são fatores de risco para doença cardiovascular, devem ser reduzidos a doses mínimas. A retirada de corticóides do esquema de imunossupressão é ainda controversa. ${ }^{31}$

\section{Controle glicêmico do paciente diabético pós-transplante renal}

O controle metabólico do paciente diabético após transplante renal oferece menor dificuldade do que na fase pré-dialítica ou dialítica. O paciente diabético com insuficiência renal crônica apresenta grandes dificuldades no manuseio da insulina e consequentemente de seu controle glicêmico, principalmente quando já se encontra em diálise. A insulina exógena é primariamente eliminada pelos rins, sendo livremente filtrada pelos glomérulos, e em seguida reabsorvida no túbulo contornado proximal, onde é parcialmente degradada por via enzimática ${ }^{32}$ Com a progressão da doença renal, o clearance de insulina diminui, com conseqüente aumento da insulina circulante. Isso se traduz, clinicamente, por uma redução da necessidade de insulina em pacientes em fase pré-dialítica ou dialítica, com difícil previsão de sua quantidade e de sua meia vida. Existem situações adicionais que agravam o controle glicêmico nesses pacientes, como aumento da resistência periférica à ação da insulina, presença de infecção e inflamação, redução do apetite causado pela subdiálise, falta de aderência às restrições dietéticas, entre outros. ${ }^{33}$

Com a recuperação da função renal que ocorre após o transplante renal, o manuseio da insulina volta a respeitar as características das diferentes formulações com relação aos picos de ação e cálculo de meia-vida, o que facilita o controle metabólico. Deve-se ressaltar que, imediatamente após o transplante renal com sucesso, a necessidade de insulina passa a ser de duas a três vezes maior do que no período de hemodiálise, como resultado da boa função renal e do uso de corticóides.

\section{EFEITO DO TRANSPLANTE RENAL SOBRE COMPLICAÇÕES DIABÉTICAS}

\section{Retinopatia}

Existem poucos relatos na literatura avaliando especificamente a evolução da retinopatia diabética após transplante de rim isolado. Após a realização do transplante renal é comum a estabilização da retinopatia: as lesões microvasculares que afetam a retina se estabilizam em $59 \%$ dos casos, melhoram em $27 \%$ e pioram em $14 \%$ dos casos. ${ }^{34}$

Embora existam algumas publicações mostrando que a evolução da retinopatia diabética é semelhante pós-transplante de rim isolado ou após transplante simultâneo de rim-pâncreas, ${ }^{35}{ }^{36}$ o estado de normoglicemia mantido após o transplante duplo com sucesso parece ter impacto mais favorável na retinopatia diabética. ${ }^{37}$ Nos diabéticos tipo 2 ocorre estabilização das lesões proliferativas e não-proliferativas em $90 \%$ dos pacientes após transplante renal. ${ }^{38}$

\section{Nefropatia}

Os pacientes diabéticos submetidos a transplante renal podem desenvolver no enxerto renal as mesmas lesões que determinaram a insuficiência renal crônica. No entanto, a incidência dessa ocorrência é relativamente pequena, sendo de 0,4 a $1,8 \%{ }^{39}$ e tardia. ${ }^{40}$ Os enxertos renais cuja biópsia revela recorrência da nefropatia diabética apresentam menor sobrevida. ${ }^{41}$ Não existem estudos que avaliaram o impacto do uso dos inibidores da enzima conversora e inibidores do receptor da angiotensina na progressão da nefropatia diabética recorrente no enxerto renal. 


\section{Neuropatia}

O transplante renal isolado tem limitado efeito sobre a neuropatia diabética sensitiva e motora. A melhora da neuropatia observada após transplante renal aparentemente se deve principalmente pela melhora do componente de neuropatia urêmica.

Com relação à neuropatia autonômica, ocorre melhora dos sintomas relacionados com a neuropatia gastrointestinal após transplante renal. ${ }^{42} \mathrm{O}$ transplante renal está também associado a uma melhora nos episódios de hipotensão postural. ${ }^{43}$

\section{Complicações cardiovasculares}

A principal causa de mortalidade nos pacientes diabéticos transplantados renais é a doença cardiovascular. Os pacientes diabéticos transplantados apresentam maior número de eventos cardiovasculares que os não-diabéticos, com incidência de $20 \%$ e $5 \%$, respectivamente. ${ }^{44}$

\section{Doença óssea}

Os pacientes diabéticos e não-diabéticos submetidos a transplante renal apresentam perda óssea acentuada no período pós-transplante, que leva a fraturas no período pós-transplante: cerca de $22,5 \%$ dos pacientes transplantados são acometidos por fraturas ósseas nos primeiros cinco anos pós-transplante, enquanto que em diabéticos o número de fraturas chega a $36 \%{ }^{45}$

\section{Qualidade de vida}

As complicações secundárias do diabetes associadas às complicações da doença renal crônica comprometem de forma muito acentuada a qualidade de vida desses pacientes. A instituição do tratamento dialítico, seja por hemodiálise ou diálise peritoneal, representa um complicador maior. Além das dificuldades referentes ao próprio procedimento, como por exemplo, dificuldade de acesso vascular para hemodiálise, aumento do número de infecções, aumento do risco cardiovascular, restrições alimentares e de ingestão hídrica, existem também as limitações relacionadas ao comprometimento da acuidade visual, da locomoção (tanto pela neuropatia ou até mesmo por amputações).

Com o transplante renal, esses pacientes passam a experimentar uma melhora significativa na qualidade de vida: ocorre melhora física, psicológica, percepção de saúde e da função social. ${ }^{43,46}$ Estudos anteriores demonstraram que pacientes com doença renal crônica submetidos a transplante renal obtiveram melhor qualidade de vida (comer, beber, atividade física, descanso, aspectos psicossociais, vida normal) em relação a pacientes mantidos em diálise. ${ }^{47}$

\section{RESULTADOS}

\section{Sobrevida do paciente diabético: diálise versus transplante renal}

Os pacientes diabéticos em programa de diálise apresentam pior evolução clínica que os não-diabéticos. ${ }^{48}$ Knoll \& Nichol ${ }^{49}$ mostraram que a expectativa de vida e a expectativa de vida ajustada à qualidade de vida foram significativamente menores em pacientes diabéticos em diálise, quando comparados a qualquer modalidade de transplante (tabela 1).
Tabela 1 - Análise da expectativa de vida e QALY (quality-adjusted life expectancy - expectativa de vida ajustada à qualidade de vida) nas diversas modalidades de tratamento para o paciente portador de DM tipo $1 \mathrm{com}$ doença renal crônica.

\begin{tabular}{|c|c|c|c|c|c|}
\hline & $\begin{array}{c}\text { Expectativa } \\
\text { de vida } \\
\text { (anos) }\end{array}$ & $\begin{array}{c}\text { QALY } 49 \\
\text { (anos) }\end{array}$ & $\begin{array}{c}\text { Sobrevida } \\
\text { do paciente } \\
7^{\circ} \text { ano }^{53}\end{array}$ & $\begin{array}{c}\text { Sobrevida } \\
\text { do paciente } \\
8^{\circ} \text { ano }\end{array}$ & $\begin{array}{c}\text { Sobrevida } \\
\text { do paciente } \\
10^{\circ} \text { ano }\end{array}$ \\
\hline $\begin{array}{c}\text { Transplante } \\
\text { de rim com } \\
\text { doador vivo }\end{array}$ & 18,3 & 10,3 & $\begin{array}{c}\text { não } \\
\text { analisado }\end{array}$ & $72 \%$ & $65 \%$ \\
\hline $\begin{array}{c}\text { Transplante } \\
\text { de rim com } \\
\text { doador } \\
\text { falecido }\end{array}$ & 11,4 & 6,5 & $56 \%$ & $55 \%$ & $46 \%$ \\
\hline $\begin{array}{c}\text { TSRP } \\
\text { TSRP }\end{array}$ & 15,7 & 9,1 & $77 \%$ & $72 \%$ & $67 \%$ \\
\hline Diálise & 7,8 & 4,5 & $40 \%$ & - & - \\
\hline
\end{tabular}

TSRP: transplante simultâneo de rim-pâncreas

Sørensen e cols mostraram que o transplante renal em pacientes diabéticos reduz em $79 \%$ o risco de morte, comparado a pacientes diabéticos mantidos em diálise que não receberam transplante renal. ${ }^{50}$ Em cinco anos, a sobrevida foi de $96 \%$ para pacientes diabéticos transplantados versus $58 \%$ para pacientes diabéticos mantidos em diálise crônica; em 10 anos, $72 \%$ contra $31 \%$, respectivamente. ${ }^{51}$

Brunkhorst e cols mostraram que a hemodiálise constitui um risco significativo de morbidade e mortalidade para pacientes diabéticos em hemodiálise, e que o transplante renal promove uma significativa melhora da sobrevida. ${ }^{52}$ Neste estudo, o risco relativo de óbito foi significativamente maior em pacientes diabéticos que permaneceram em diálise do que pacientes diabéticos submetidos a transplante (odds ratio $=0,29$ ).

\section{Transplante de rim isolado: sobrevida do paciente diabético versus não-diabético}

Os resultados de transplante renal em pacientes diabéticos são inferiores quando comparados a pacientes não-diabéticos.

Existe risco de morte $88 \%$ maior em pacientes diabéticos submetidos a transplante renal em relação a pacientes não-diabéticos submetidos ao mesmo procedimento. ${ }^{50}$

Como apresentado acima, pacientes diabéticos apresentam elevado risco cardiovascular. Esse elevado risco se traduz em menor sobrevida no paciente transplantado em relação a pacientes nãodiabéticos, com maior mortalidade por causas cardíacas, infecciosas e mortes sem causa atribuída. ${ }^{44}$

Sørensen e cols analisaram as taxas de sobrevida de pacientes transplantados renais portadores de diabetes mellitus (tipo 1 e 2) em comparação aos não-diabéticos. No $1^{\circ}$ ano pós-transplante, a sobrevida do paciente foi de 95 versus $93 \%$, em cinco anos de 80 versus $85 \%$ e em dez anos de 52 versus $71 \%$ para pacientes diabéticos versus não-diabéticos, respectivamente..$^{50}$

Os resultados mais recentes disponíveis pelo registro OPTN (Organ Procurement and Transplantation Network) (tabela 2) mostram dados relativos ao transplante renal realizado em pacientes diabéticos (tipo 1 e tipo 2) comparados ao registro geral de pacientes submetidos a transplante renal (incluindo também os pacientes diabéticos). 
Tabela 2 - Dados de sobrevida do paciente e enxerto (rim e pâncreas) nas diversas modalidades de transplante para o paciente diabético, obtidos da OPTN (Organ Procurement and Transplantation Network). ${ }^{2}$

\begin{tabular}{|c|c|c|c|c|c|c|}
\hline \multirow{2}{*}{$\begin{array}{l}\text { Modalidade de } \\
\text { transplante }\end{array}$} & \multicolumn{2}{|c|}{ Paciente } & \multicolumn{2}{|c|}{$\begin{array}{l}\text { Enxerto } \\
\text { RIM }\end{array}$} & \multicolumn{2}{|c|}{$\begin{array}{c}\text { Enxerto } \\
\text { PÂNCREAS }\end{array}$} \\
\hline & 1 ano & 5 anos & 1 ano & 5 anos & 1 ano & 5 anos \\
\hline $\begin{array}{c}\text { Tx Rim } \\
\text { (doador vivo) }\end{array}$ & $98,5 \%$ & $91,4 \%$ & $95,5 \%$ & $81,5 \%$ & - & - \\
\hline $\begin{array}{c}\text { Tx Rim em diabéticos } \\
\text { (doador vivo) }\end{array}$ & $96,8 \%$ & $83,1 \%$ & $95,1 \%$ & $76,3 \%$ & - & - \\
\hline $\begin{array}{c}\text { Tx Rim* } \\
\text { (doador falecido SCE) }\end{array}$ & $95,4 \%$ & $84,5 \%$ & $91,7 \%$ & $72,7 \%$ & - & - \\
\hline $\begin{array}{l}\text { Tx Rim em diabéticos } \\
\text { (doador falecido SCE) }\end{array}$ & $93,3 \%$ & $72,5 \%$ & $89,7 \%$ & $65,3 \%$ & - & - \\
\hline $\begin{array}{c}\text { Tx Rim* } \\
\text { (doador falecido CE) }\end{array}$ & $90,6 \%$ & $71,9 \%$ & $84,8 \%$ & $59,7 \%$ & - & - \\
\hline $\begin{array}{l}\text { Tx Rim em diabéticos } \\
\text { (doador falecido CE) }\end{array}$ & $87,8 \%$ & $60,3 \%$ & $82,3 \%$ & $49,5 \%$ & - & - \\
\hline TSRP & $94,8 \%$ & $86,5 \%$ & $92 \%$ & $78,9 \%$ & $84,2 \%$ & $73,3 \%$ \\
\hline Tx Pâncreas após-Rim & $96,3 \%$ & $83,8 \%$ & - & - & $77,6 \%$ & $55,4 \%$ \\
\hline Tx Pâncreas Isolado & $97,6 \%$ & $88,7 \%$ & - & - & $75,3 \%$ & $52,7 \%$ \\
\hline
\end{tabular}

Fonte: OPTN (Organ Procurement and Transplantation Network / Scientific Registry of Transplant Recipients (http://optn.transplant.hrsa.gov/) dados disponiveis em junho de 2010). Dados sem ajuste.

$T x=$ transplante

TSRP: transplante simultâneo de rim-pâncreas

* = resultados incluíram todos os pacientes, não-diabéticos e diabéticos SCE $=$ sem critério expandido

$C E=$ critério expandido

\section{Transplante de rim isolado para paciente diabético: doador vivo versus doador falecido}

Diversos estudos baseados na análise da sobrevida do paciente e do enxerto assim como da qualidade de vida demonstraram que em pacientes diabéticos tipo 1 o transplante de rim isolado com doador vivo oferece melhores resultados do que o transplante renal com doador-falecido (tabela 1)..$^{53-55}$

Segundo dados da OPTN (tabela 2), a análise do transplante renal com doador vivo no subgrupo de pacientes diabéticos $(n=8.094)$ mostrou resultados inferiores tanto de sobrevida do paciente como do enxerto, principalmente em longo prazo (cinco anos póstransplante), quando comparados a todos os pacientes submetidos a transplante de rim com doador vivo $(n=30.237)$. Após um e cinco anos, a sobrevida do paciente foi de $96,8 \%$ e $83,1 \%$ para transplante realizado com doador vivo, e a sobrevida do enxerto após um e cinco anos foi de $95,1 \%$ e $76,3 \%$.

Os piores resultados do transplante em pacientes diabéticos são observados quando o transplante renal foi realizado com doador falecido. Após um e cinco anos, a sobrevida do paciente foi de $93,3 \%$ e $72,5 \%$ para transplante realizado com doador falecido sem critério expandido e a sobrevida do enxerto após um e cinco anos foi de $89,7 \%$ e $65,3 \%$ para enxertos de doadores falecidos sem critério expandido. Os pacientes submetidos a transplante renal isolado com doador falecido com critério expandido obtiveram os piores resultados de sobrevida, comparado aos transplantados com doadores vivos e doadores falecidos sem critério expandido.

\section{Transplante para paciente diabético: transplante de rim} isolado versus transplante simultâneo de rim-pâncreas

A análise de sobrevida tardia do paciente revela superioridade do transplante de rim isolado com doador vivo e do transplante de pâncreas-rim simultâneo sobre o transplante de rim isolado com doador cadáver. ${ }^{54,2,56-59}$

Os resultados da análise de expectativa de vida e expectativa de vida ajustada à qualidade de vida (QALY - quality-adjusted life expectancy) demonstram que o transplante de rim com doador vivo apresenta melhores resultados, seguido pelo transplante de pâncreas-rim simultâneo e transplante de pâncreas após rim. ${ }^{49}$

Segundo dados da OPTN, a sobrevida do paciente diabético submetido a transplante renal com doador vivo ou transplante simultâneo rim e pâncreas é semelhante nas duas modalidades. Após um e cinco anos, a sobrevida do paciente foi de $96,8 \%$ e $83,1 \%$ para transplante realizado com doador vivo e $94,8 \%$ e $86,5 \%$ para transplante simultâneo de rim e pâncreas. A sobrevida do enxerto renal também é semelhante nas duas modalidades de transplante (tabela 2). ${ }^{2}$

Na tabela 3 estão alguns estudos publicados na literatura, onde foram analisadas as diversas modalidades de transplante para paciente diabético tipo 1: transplante renal isolado com doador vivo ou falecido e transplante simultâneo de rim-pâncreas. Todos os estudos apresentam resultados semelhantes, ao concluírem que a pior sobrevida tanto do paciente quanto do enxerto é obtida quando se realiza transplante renal isolado com doador falecido. Nas duas opções restantes, transplante renal isolado com doador vivo ou transplante duplo, a sobrevida do paciente e do enxerto renal é semelhante. Recentemente, foi estudado o impacto que a função pancreática tem na sobrevida do paciente e do enxerto renal, uma vez que haveria melhor controle metabólico, melhora na qualidade de vida e o paciente não estaria exposto aos efeitos tóxicos crônicos da hiperglicemia. Foi demonstrado que a longo prazo, pacientes submetidos a transplante duplo que apresentam enxerto pancreático funcionante obtiveram melhor sobrevida em relação aos pacientes submetidos a transplante renal isolado ou transplante duplo em que a função pancreática foi perdida (tabela 3). ${ }^{60}$

Tabela 3 - Sobrevida do paciente e do enxerto (rim e pâncreas) nas diversas modalidades de transplante para pacientes diabéticos, disponíveis em publicações.

\begin{tabular}{|c|c|c|c|c|c|c|c|c|}
\hline \multirow{2}{*}{ Autor } & \multirow{2}{*}{$\begin{array}{l}\text { Modalidade } \\
\text { de } \\
\text { transplante }\end{array}$} & \multirow{2}{*}{$n$} & \multicolumn{2}{|c|}{ Paciente } & \multicolumn{2}{|c|}{$\begin{array}{c}\text { Enxerto } \\
\text { RIM }\end{array}$} & \multicolumn{2}{|c|}{$\begin{array}{c}\text { Enxerto } \\
\text { PÂNCREAS }\end{array}$} \\
\hline & & & 1 ano & 5 anos & 1 ano & 5 anos & 1 ano & $\begin{array}{c}5 \\
\text { anos }\end{array}$ \\
\hline \multirow{4}{*}{ Raydill et al ${ }^{56}$} & $\begin{array}{c}\text { Tx Rim em } \\
\text { DM } 1 \\
\text { (doador vivo - } \\
\text { HLA idêntico) }\end{array}$ & 46 & $100 \%$ & $94 \%$ & $96 \%$ & $85 \%$ & - & - \\
\hline & $\begin{array}{c}\text { Tx Rim em } \\
\text { DM } 1 \\
\text { (doador vivo - } \\
\text { haplo) }\end{array}$ & 84 & $99 \%$ & $85 \%$ & $94 \%$ & $72 \%$ & - & - \\
\hline & $\begin{array}{l}\text { Tx Rim em } \\
\text { DM 1 } \\
\text { (doador } \\
\text { falecido) }\end{array}$ & 296 & $94 \%$ & $72 \%$ & $86 \%$ & $64 \%$ & - & - \\
\hline & TSRP & 379 & $96 \%$ & $88 \%$ & $87 \%$ & $78 \%$ & $86 \%$ & $74 \%$ \\
\hline
\end{tabular}




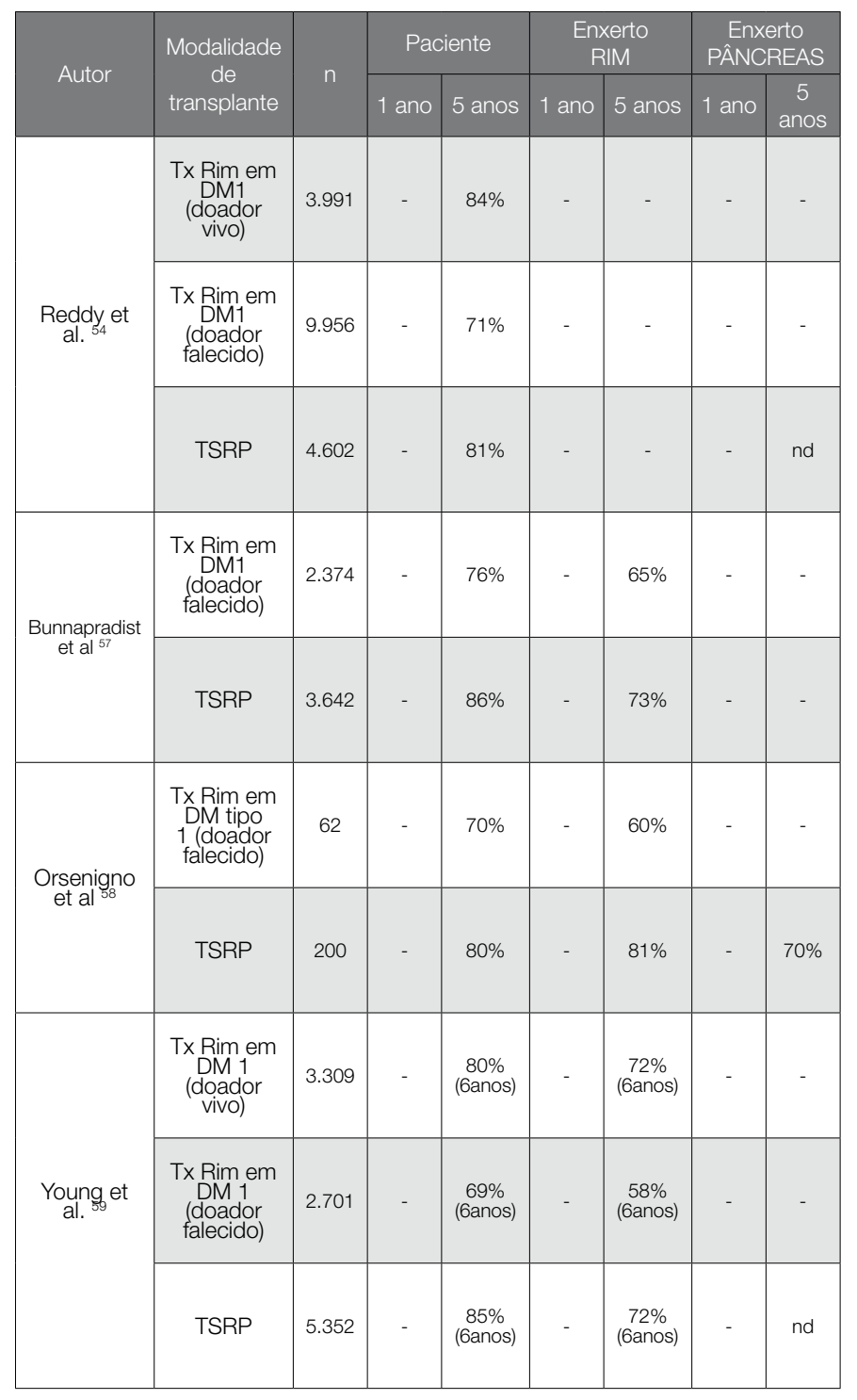

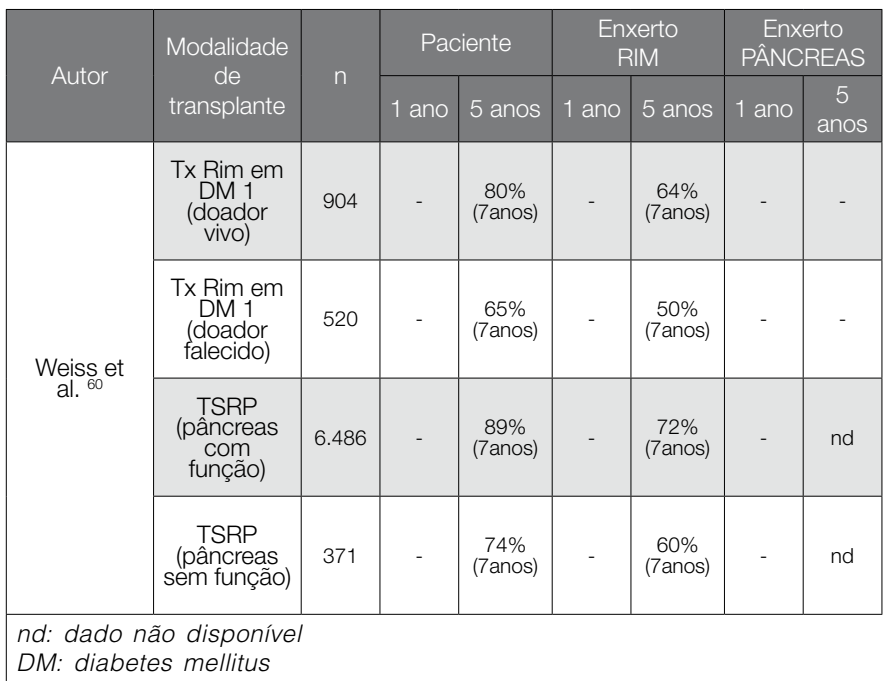

Em resumo, para pacientes com DM tipo 1, os resultados do transplante de rim isolado com doador vivo são comparáveis aos de transplante simultâneo de rim-pâncreas, estando esse particularmente indicado em casos com complicações progressivas do DM e casos que não tenham doador vivo disponível.

\section{CONCLUSÃO}

Para paciente diabético com doença renal crônica em estágio V, o transplante renal é a melhor opção terapêutica. Aos candidatos a esse tipo de tratamento, recomenda-se uma avaliação criteriosa da doença cardiovascular e das disfunções miccionais, devido à alta prevalência nesses doentes. Após transplante renal, observa-se estabilização da retinopatia e melhora na qualidade de vida, nos episódios de hipotensão postural e sintomas gastrointestinais. No entanto, o número de eventos cardiovasculares é superior aos dos não-diabéticos e, além disso, pode ocorrer recidiva da nefropatia no enxerto. Preconiza-se a realização de transplante renal com doador vivo ou transplante duplo rim e pâncreas de doador falecido pelos melhores resultados observados com essas modalidades.

\section{ABSTRACT}

Diabetes mellitus is one of the major causes for chronic kidney disease in Brazil and worldwide. Chronic kidney disease stage V patients require renal replacement therapy: dialysis or kidney transplant. Although the majority of patients are on dialysis, this modality of treatment is associated to the lowest patient survival. Transplantation options for these patients include kidney transplantation with living donor, deceased donor or simultaneous kidney-pancreas transplantation. The aim of this study was to make a review on specific aspects of kidney transplantation in diabetic patients, to assess the impact of kidney transplantation on chronic complications of diabetes, and finally to show which would be the best transplantation modality for those patients. Concerning pre-transplant aspects, due to the high cardiovascular risk in those patients, the assessment of the coronary artery disease and peripheral arterial disease should be carried out in more details in the diabetic patient. Moreover, the study of miccional dysfunction is indicated due to the high prevalence of neurogenic bladder in those patients.

As to chronic complications of diabetes, kidney transplantation can induce a stabilization of retinal lesions in most patients, improvement in the quality of life, improvement of the episodes of postural hypotension and gastrointestinal symptoms. With regard to transplant modalities available for diabetic patients, the best results for the patient and graft survival were attained by isolated kidney transplantation from living donor or simultaneous kidney-pancreas transplantation. The modality of kidney transplantation with deceased donor was associated to the worse survival rate both for patient and graft.

Keywords: Diabetes Mellitus; Renal Insufficience; Transplantation. 


\section{REFERÊNCIAS}

1. ABTO (Associação Brasileira de Transplante de Órgãos). [ Acesso em 16 jun 2010]; Disponível em HTTP://www.abto.org.br.

2. OPTN (Organ Procurement and Transplantation Network / Scientific Registry of Transplant Recipients). [Acesso em 16 jun 2010]; Disponível em $<\mathrm{http} / / /$ optn. transplant.hrsa.gov>.

3. K/DOQI clinical practice guidelines for chronic kidney disease: evaluation, classification, and stratification. Kidney Disease Outcome Quality Initiative. Am J Kidney Dis. 2002;39:S1-S246.

4. Martínez-Ramírez H, Jalomo-Martínez B, Cortés-Sanabria L, Rojas-Campos E, Barragán G, Alfaro G, et al. Renal Function Preservation in Type 2 Diabetes Mellitus Patients With Early Nephropathy: A Comparative Prospective Cohort Study Between Primary Health Care Doctors and a Nephrologist. Am J Kidney Dis. 2006;47:78-87.

5. Becker BN, Rush SH, Dykstra DM, Becker YT, Port FK. Preemptive transplantation for patients with diabetes-related kidney disease. Arch Intern Med. 2006;166:44-8

6. Krolewski AS, Kosinski EJ, Warram JH, Leland OS, Busick EJ, Asmal AC, et al. Magnitude and determinants of coronary artery disease in juvenile-onset, insulindependent diabetes mellitus. Am J Cardiol. 1987;59:750-5.

7. Okuno Y, Sato K. Diabetes mellitus and vascular calcification. Clin Calcium. 2002;12:1094-101.

8. Sarnak MJ, Levey AS. Cardiovascular disease and chronic renal disease: A New Paradigm. Am J Kidney Dis. 2000;35:S117-S131.

9. Ohtake T, Kobayashi S, Moriya $\mathrm{H}$, et al. High prevalence of occult coronary artery stenosis in patients with chronic kidney disease at the initiation of renal replacement therapy: An angiographic examination. J Am Soc Nephrol. 2005;16:1141-8.

10. Coelho JC, Siqueira R M, Farret TCF, Fritsch M, Fedrizzi R, Goldbaum M, et al. Prevalência de doença arterial coronariana em candidatos a transplante simultâneo de pâncreas e rim. J Bras Transpl. 2007;10:789-840.

11. Ramanathan V, Goral S, Tanriover B, et al. Screening asymptomatic diabetic patients for coronary artery disease prior to renal transplantation. Transplantation. 2005;79:1453-8

12. De Lima JJG, Sabbaga E, Vieira MLC, Paula FJ, Ianhez LE, Krieger E, Ramires JAF. Coronary Angiography Is the Best Predictor of Events in Renal Transplant Candidates Compared With Noninvasive Testing. Hypertension 2003;42:263-268.

13. Kasiske BL, Ramos EL, Gaston RS, et al. The evaluation of renal transplant candidates: clinical practice guidelines. J Am Soc Nephrol. 1995;6:1-34.

14. Mautone A, Brown JR. Contrast-Induced Nephropathy in Patients Undergoing Elective and Urgent Procedures. J Interv Cardiol. 2010;23:78-85.

15. Thachil J, Gatt A, Martlew V. Management of surgical patients receiving anticoagulation and antiplatelet agents. Br J Surg. 2008;95:1437-48

16. Almdal T, Scharling H, Jensen JS, Vestergaard H. The Independent Effect of Type 2 Diabetes Mellitus on Ischemic Heart Disease, Stroke, and Death A PopulationBased Study of 13000 Men and Women With 20 Years of Follow-up. Arch Intern Med. 2004;164:1422-6.

17. Selvin E, Erlinger TP. Prevalence of and risk factors for peripheral arterial disease in the united states results from the national health and nutrition examination survey, 1999-2000. Circulation. 2004;110:738-43.

18. Sadowski EA, Bennett LK, Chan MR, Wentland AL, Garrett AL, Garrett RW, et. al. Nephrogenic Systemic Fibrosis: Risk Factors and Incidence Estimation. Radiology 2007;243:148-157.

19. Boulton AJM. The diabetic foot: grand overview, epidemiology and pathogenesis Diabetes Metab Res Rev. 2008;24:S3-S6.

20. Akbari CM, LoGerfo FW. Diabetes and peripheral vascular disease. J Vasc Surg. 1999;30:373-4.

21. Daneshgari F, Liu G, Birder L, Hanna-Mitchell AT, Chacko S. Diabetic bladder dysfunction: current translational knowledge. J Urol. 2009;182 S18-S26.

22. Geerlings, SE. Urinary tract infections in patients with diabetes mellitus: epidemiology, pathogenesis and treatment. Int J Antimicrob Agents. 2008;31: S54-S57.

23. Bunnapradist S, Danovitch GM. Evaluation of adult kidney transplant candidates. Am J Kidney Dis. 2007;50:890-8.

24. Chakkera HA, Weil JE, Castro J, Heilman RL, Reddy KS, Mazur MJ, et al. Hyperglycemia during the immediate period after kidney transplantation. Clin J Am Soc Nephrol. 2009;4:853-9.

25. Ligtenberg G, Hené RJ, Blankestijn PJ, Koomans HA. Cardiovascular risk factors in renal transplant patients: cyclosporin A versus tacrolimus. J Am Soc Nephrol. 2001;12:368-73.

26. Hardinger KL, Brennan DC, Lowell J, Schnitzler MA. long-term outcome of gastrointestinal complications in renal transplant patients treated with mycophenolate mofetil. Transplant Int. 2004;17:609-16.

27. Johnston O, Rose CL, Webster AC, Gill JS. Sirolimus Is Associated with New-Onset Diabetes in Kidney Transplant Recipients. J Am Soc Nephrol. 2008;19:1411-8.

28. Cardinal H, Froidure A, Dandavino R, Daloze P, Hébert MJ, Colette S, Boucher A. Conversion From Calcineurin Inhibitors to Sirolimus in Kidney Transplant Recipients: A Retrospective Cohort Study. Transplant Proc. 2009;41:3308-10

29. Schena FP, Pascoe MD, Alberu J, Rial MC, Oberbauer R, Brennan DC, Campistol JM, et. al. Conversion From Calcineurin Inhibitors to Sirolimus Maintenance Therapy in Renal Allograft Recipients: 24-Month Efficacy and Safety Results From the CONVERT Trial. Transplantation 2009;87:233-242.

30. P. Matias, M.R. Araujo, J.E. Romão Jr, H. Abensur, and I.L. Noronha. Conversion to Sirolimus in Kidney-Pancreas and Pancreas Transplantation. Transplant Proc. 2008;40:3601-5.

31. Pascual J, Galeano C, Royuela A, Zamora J. A Systematic Review on Steroid Withdrawal Between 3 and 6 Months After Kidney Transplantation. Transplantation 2010;90:343-9.

32. Reilly JB, Berns JS. Selection and dosing of medications for management of diabetes in patients with advanced kidney disease. Semin Dial. 2010;23:163-8.

33. ShriShrimal $\mathrm{K}$, Hart $\mathrm{P}$, Michota F. Managing diabetes in hemodialysis patients: observations and recommendations. Cleve Clin J Med. 2009;76:649-55.

34. Laatikainen L, Summanen P, Ekstrand A, Groop L. Ophthalmological follow-up of diabetic patients after kidney transplantation. Ger J Ophthalmol. 1993;2:24-7.

35. Scheider A, Meyer-Schwickerath E, Nusser J, Land W, Landgraf R. Diabetic retinopathy and pancreas transplantation: a 3-year follow-up. Diabetologia. 1991;34:S95-9.

36. Wang Q, Klein R, Moss SE, Klein BE, Hoyer C, Burke K, et al. The influence of combined kidney-pancreas transplantation on the progression of diabetic retinopathy. A case series. Ophthalmology. 1994;101:1071-6.

37. Koznarova R, Saudek F, Sosna T, Adamec M, Jedinakova T, Boucek P, et al. Beneficial effect of pancreas and kidney transplantation on advanced diabetic retinopathy. Cell Transplant. 2000;9: 903-8.

38. Mittal NS, Bajwa GS, Sandhu JS. Diabetic retinopathy before and after renal transplantation: a longitudinal study. Transplant Proc. 2005;37:2077-9.

39. Salifu MO, Nicastri AD, Markell MS, Ghali H, Sommer BG, Friedman EA. Allograft diabetic nephropathy may progress to end-stage renal disease. Pediatr Transplantation. 2004;8:351-6.

40. Bhalla V, Nast CC, Stollenwerk N, Tran S, Barba L, Kamil ES, et al. Recurrent and de novo diabetic nephropathy in renal allografts. Transplantation. 2003;75:66-71.

41. Hariharan S, Smith RD, Viero R, First MR. Diabetic nephropathy after renal transplantation. Transplantation. 1996;62:632-5.

42. Hathaway D, Abell T, Cardoso S, Hartwig M, Elmer D, Horton J, et al. Improvement in 
autonomic function following pancreas-kidney versus kidney-alone transplantation. Transplant Proc. 1993;25:1306-8.

43. Gaber AO, Hathaway DK, Abell T, Cardoso S, Hartwig MS, Gebely S. Improves autonomic and gastric function in pancreas - kidney versus kidney-alone transplantation contributes to quality of life. Transplant Proc. 1994;26:515-6.

44. Snyder JJ, Kasiske BL, Maclean R. Peripheral arterial disease and renal transplantation. J Am Soc Nephrol. 2006;17:2056-68.

45. Nikkel LE, Hollenbeak CS, Fox EJ, Uemura T, Ghahramani N. Risk of fractures after renal transplantation in the United States. Transplantation 2009;87:1846-51.

46. Zehrer CL, Gross CR. Comparison of quality of life between pancreas/kidney and kidney transplant recipients: 1-Year Follow-up. Transplant Proc. 1994;26:508-9.

47. Apostolou T, Hutchison AJ, Boulton AJ, Chak W, Vileikyte L, Uttley L, et al. Quality of life in CAPD, transplant, and chronic renal failure patients with diabetes. Ren Fail. 2007;29:189-97.

48. Wolfe RA, Ashby VB, Milford EL, Ojo AO, Ettenger RE, Agodoa LY, et al Comparison of mortality in all patients on dialysis, patients on dialysis awaiting transplantation, and recipients of a first cadaveric transplant. N Engl J Med. 1999;341:1725-30

49. Knoll GA, Nichol G. Dialysis, kidney transplantation, or pancreas transplantation for patients with diabetes mellitus and renal failure: a decision analysis of treatment options. J Am Soc Nephrol. 2003;14:500-15

50. Sørensen VR, Mathiesen E R, Heaf J, Feldt-Rasmussen B. Improved survival rate in patients with diabetes and end-stage renal disease in Denmark. Diabetologia. 2007;50:922-9.

51. Sato S, Babazono T, Tomonaga O, Nakamura M, Hoshino T, Sageshima J, et al Clinical study of kidney transplantation in diabetic patients with end-stage renal disease. Transplant Proc. 1998;30:3097-8.

52. Brunkhorst R, Lufft V, Dannenberg B, Kliem V, Tusch U, Pichlmayr R. Improved survival in patients with type 1 diabetes mellitus after renal transplantation compared with hemodialysis: A case-control study. Transplantation. 2003;76:115-9.

53. La Rocca E, Fiorina P, di Carlo V, Astorri E, Rossetti C, Lucignani G, et al. Cardiovascular outcomes after kidney-pancreas and kidney-alone transplantation. Kidney Int. 2001;60:1964-71.

54. Reddy KS, Stablein D, Taranto S, Stratta RJ, Johnston TD, Waid TH, et al. Longterm survival following simultaneous kidney-pancreas transplantation versus kidney transplantation alone in patients with type 1 diabetes mellitus and renal failure. Am J Kidney Dis. 2003;41:464-70.

55. Ojo AO, Meier-Kriesche H, Hanson JA, Leichtman A, Magee JC, Cibrik D, et al. The impact of simultaneous pancreas-kidney transplantation on long-term patient survival. Transplantation. 2001;71:82-90.

56. Rayhill SC, D’Alessandro AM, Odorico JS, Knechtle SJ, Pirsch JD, Heisey DM, et al. Simultaneous pancreas-kidney transplantation and living related donor renal transplantation in patients with diabetes: is there a difference in survival?. Ann Surg. 2000;231:417-23.

57. Bunnapradist S, Cho YW, Cecka JM, Wilkinson A, Danovitch GM. Kidney allograft and patient su5rvival in type I diabetic recipients of cadaveric kidney alone versus simultaneous pancreas/kidney transplants: a multivariate analysis of the UNOS database. J Am Soc Nephrol. 2003;14:208-13.

58. Orsenigo E, Socci C, Fiorina P, Zuber V, Secchi A, Di Carlo V, et al. Cardiovascular benefits of simultaneous pancreas-kidney transplant versus kidney alone transplant in diabetic patients. Transplant Proc. 2005;37:3570-1.

59. Young BY, Gill J, Huang E, Takemoto SK, Anastasi B, Shah T, et al. Living donor kidney versus simultaneous pancreas-kidney transplant in type I diabetics: an analysis of the OPTN/UNOS database. Clin J Am Soc Nephrol. 2009;4:845-52.

60. Weiss AS, Smits G, Wiseman AC. Twelve-month pancreas graft function significantly influences survival following simultaneous pancreas- kidney transplantation. Clin J Am Soc Nephrol. 2009;4:988-95. 